\title{
Patterns of exposure to adverse childhood experiences and their associations with mental health: a survey of 1346 university students in East Asia
}

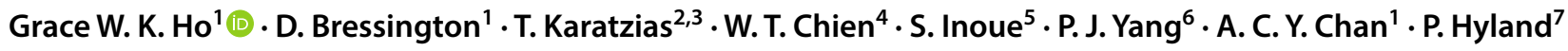

Received: 1 April 2019 / Accepted: 3 September 2019 / Published online: 9 September 2019

(c) Springer-Verlag GmbH Germany, part of Springer Nature 2019

\begin{abstract}
Introduction Adverse childhood experiences (ACEs) constitute a significant global mental health burden. Prior studies typically investigated the impact of ACEs on mental health using a cumulative risk approach; most ACEs studies were also conducted in Western settings.

Purpose This study aimed to examine ACEs using a pattern-based approach and assess their associations with mental health outcomes by early adulthood in East Asia.

Methods The present study included measures of exposure to 13 categories of ACEs, depression, anxiety, maladjustment, and posttraumatic stress in a sample of 1346 university students from Hong Kong, China, Taiwan, and Japan.

Results Latent class analysis indicated three distinct patterns of ACE exposure: Class 1: Low ACEs (76.0\%); Class 2: Household Violence (20.6\%); and Class 3: Household Dysfunction (3.4\%). Those representing Class 3 had significantly more ACEs compared with those in Classes 1 or 2 . Controlling for age and sex, those in Class 2 reported significantly higher depression and maladjustment symptoms compared with those in Class 1; both Classes 2 and 3 had significantly higher anxiety symptoms and odds for meeting diagnostic criteria for posttraumatic stress disorders compared with those in Class 1 . Conclusions Study findings suggest that young adults' mental health, at least under certain contexts, is more closely linked with the nature and pattern of ACE co-occurrence, rather than the number of ACEs.
\end{abstract}

Keywords Adverse childhood experiences $\cdot$ East Asia $\cdot$ Latent class analysis $\cdot$ Mental health $\cdot$ Young adults

Grace W. K. Ho

grace.wk.ho@polyu.edu.hk

1 School of Nursing, The Hong Kong Polytechnic University, PQ426, Hung Hom, Hong Kong

2 School of Health and Social Care, Edinburgh Napier University, Edinburgh, UK

3 NHS Lothian, Rivers Centre for Traumatic Stress, Edinburgh, UK

4 The Nethersole School of Nursing, The Chinese University of Hong Kong, Sha Tin, Hong Kong

5 Department of Nursing Science, Okayama Prefectural University, Okayama, Japan

6 Graduate Institute of Social Work, National Chengchi University, Taipei City, Taiwan

7 Department of Psychology, Maynooth University, Maynooth, Ireland

\section{Introduction}

Adverse childhood experiences (ACEs), including maltreatment, violence exposure, and other forms of serious household dysfunction in the first 18 years of life, often co-occur and confer subadditive negative impacts on mental health across the lifespan [1-3]. Ample studies have highlighted the gradated impacts of ACE exposure on mental health using a unidimensional, cumulative risk approach (i.e., the ACE score) $[4,5]$. Some emerging research using a person-centered, pattern-based approach has demonstrated discernable patterns of ACE co-occurrence that associated with different risk profiles for poorer outcomes [6-8], but these findings were largely restricted to Western contexts. Our recent study of 433 young adults from Hong Kong has uncovered a unique pattern of ACEs that is marked by exposures to high levels of interpersonal violence within the home (i.e., co-occurrence of emotional abuse, physical abuse, and witnessing domestic violence) in the absence of other serious 
household dysfunction [9]. This unique pattern of exposure had not been previously identified using Western samples, which lend evidence to support how idiosyncrasies in ACE exposure may be driven by geographic, social, and cultural norms. However, it remains unclear whether this pattern of exposure is representative of the wider East Asian culture, and how responses to different patterns of exposure may vary within this specific context. As an extension of our prior work, the present study aims to validate the patterns of ACE exposure that we previously identified in Hong Kong [9] using additional samples of young adults recruited from China, Taiwan, and Japan. The relationships between current mental health outcomes (i.e., depression, anxiety, maladjustment, and posttraumatic stress) with patterns of ACE exposure were also examined.

\section{ACEs in East Asia}

ACEs are pervasive and constitute a significant global public health concern $[4,10]$. In East Asia, defined as an ethnocultural region sharing strong Confucian societal values [11], prior country-specific studies using non-clinical samples showed that $32 \%$ of respondents in Japan [12], 50\% in South Korea [13], 74\% in Hong Kong [9], and 76\% in Vietnam [14] had at least one ACE; several studies conducted in mainland China also reported rates varying between 31 and 94\% [15-17]. Although how ACEs were measured varied considerably across studies, these findings indicate that the rates of ACE exposure may be higher in this cultural context compared with international norms. For example, the WHO World Mental Health Survey of over 50,000 adults in 21 countries found that $39 \%$ of respondents reported at least one ACE [4]. A possible driver for the higher rates of ACEs in East Asia may be due to culturally ingrained beliefs, such as those that support patriarchal norms and normative use of physical force in the home [18, 19], which may disproportionately heighten risks for certain ACEs. Indeed, a study of over 1,300 young adults from Europe and East Asia (i.e., China, Japan, and South Korea) found that East Asian respondents were more likely to endorse patriarchal beliefs and attitudes (e.g., male dominance), and reported significantly more severe interpersonal physical assault and emotional aggression perpetrated against an intimate partner compared with their European counterparts [20]. Separately, the normative use of physical force against children (e.g., corporal punishment) in East Asia may also increase risks for abuse and neglect, as the line between reasonable punishment and maltreatment is subjective and nuanced by cultural practices [21, 22]. Taken together, these findings suggest that cultural norms are closely linked with violence approval, normalization, and perpetration (e.g., child maltreatment and domestic violence), and may explain the higher rates of overall ACE exposure found in East Asian societies. However, little research has examined how ACEs tend to co-occur under this cultural context, or the outcomes associated with unique patterns of exposure.

\section{Patterns of ACE exposure}

Although ample research has documented the gradated relationships between the number of ACEs (i.e., the ACE score) with poorer physical, mental, and behavioral health outcomes [5], recent studies are beginning to adopt a person-centered approach using latent class analysis (LCA) to uncover distinct patterns of ACE co-occurrence within a given group. This emerging body of science was driven by a recognition of the methodological and conceptual limitations in understanding ACEs using a cumulative risk approach [23], which negates the contextual heterogeneity of ACE exposure and their potential associations with different health risk profiles. However, most of these studies have only been conducted in Western settings. For example, McLafferty et al. [24] identified three patterns of ACE exposure from a representative sample of 1,986 adults in Northern Ireland-'Economic adversity' (8\%), 'Polyadversity' (6\%), and 'Low risk' (86\%). Another study of over 34,000 Canadian adults found five patterns of ACE exposure- 'Caregiver substance use and maltreatment acts of omission (14\%), 'Physical and emotional maltreatment' (7\%), 'Severe cross-subtype maltreatment and caregiver substance use' (4\%), 'Caregiver maltreatment' (2\%), and 'Low adversity risk' (72\%) [8]. In the United States, four studies using large, non-clinical samples to examine typologies of ACE exposure involving both interpersonal violence (i.e., maltreatment and domestic violence) and other forms of serious household dysfunction $[6,7,23,25]$ unanimously identified two typologies of exposure represented by normative/low risk and high/multiple ACEs. In addition, patterns of exposure related exclusively to emotional ACEs, sexual abuse, household dysfunction/community violence, or a combination of maltreatment with caregiver substance use were identified.

To date, little is known about the different constellations of ACE exposure outside of Western contexts. To our knowledge, four studies have been conducted in Asia using a pattern-based approach to identify typologies of childhood victimization, two of which focused on specific subtypes of ACEs (i.e., maltreatment or household profiles). In India, Charak and Koot [26] identified four patterns of maltreatment exposure from a sample of 702 adolescents, namely 'Moderate-severe abuse and physical neglect' (16\%), 'Low to moderate-severe abuse' (30\%), 'Moderate-severe neglect' (25\%), and 'Minimal abuse or neglect' (29\%). Separately, findings from 440 children with confirmed allegations of maltreatment in Singapore uncovered four distinct household profiles-'Large household' (27\%), 'Harsh parenting' 
(27\%), 'High criminality' (12\%), and 'Low disadvantage' (34\%) [27]. Recently, a secondary analysis of data from the China Health and Retirement Longitudinal Study identified three patterns of childhood adversities-"normal childhood," "low childhood socio-economic status and health class," and "traumatic childhood" [28]. However, this study only included seven types of childhood adversities and was restricted to an older adult sample.

Our prior analysis of ACEs among 433 Hong Kong young adults [9] was the first to utilize a pattern-based approach to examine typologies of childhood adversity using a young adult sample in East Asia that included the 13 categories of ACEs as recommended by the WHO [29]. This study identified a unique pattern marked by high probabilities of exposure to household violence (i.e., abuse and witnessing domestic violence) in the absence of other serious household dysfunction, which had not been previously reported in studies conducted in Western settings. Indeed, it is suggested that many forms of serious household dysfunction, such as family member substance use, marriage dissolution, and community violence, are comparatively rare in conservative Asian cultures [30]. Since the definitions, occurrence, and co-occurrences of ACEs likely vary across geographic and sociocultural norms [4, 31], further investigations are needed to help validate patterns of ACE exposure within specific cultural contexts to deepen our understanding of the etiology and prevention of ACEs across different settings.

\section{ACEs pattern and mental health in young adulthood}

ACEs contribute significantly to the global mental health disease burden, and were estimated to account for $30 \%$ of all mental disorders worldwide [4]. It is particularly important to examine mental health outcomes associated with ACEs in young adults, because they often manifest by late adolescence and persist into early adulthood [32, 33]. However, some findings suggest that young adults' mental health is more closely linked with the severity, not the total number, of ACEs [34], thus supporting the use of a person-centered, pattern-based approach to assess the risks of negative mental health outcomes associated with unique typologies of exposure. Indeed, one study found that ACE patterns were most predictive of mental health outcomes compared with other problems in young adults, such as poorer physical health, alcohol use, and academic performance [25]. Other studies conducted in Western settings also demonstrated disparate mental health risk profiles based on patterns of ACE exposure, including anxiety, depression, substance use disorders, and suicidality [7, 23-25, 35, 36].

Although ample evidence supports the enduring relationships between different forms of childhood adversities with negative mental health outcomes in East Asia [12,
37, 38], a significant gap remains in understanding how different ACE patterns may associate with different mental health risk profiles in this cultural group. Addressing this knowledge gap would have significant implications for the design and implementation of targeted and culturally appropriate prevention and intervention programs. The purpose of this study was to validate the ACE patterns previously identified in Hong Kong [9] with additional samples of young adults from China, Taiwan, and Japan to generate representative patterns of ACE exposure in East Asia. As an extension of our prior work, the relationships between ACE patterns and four mental health outcomes (i.e., depression, anxiety, maladjustment, and posttraumatic stress) were also examined.

\section{Methods}

\section{Participants and procedures}

Participants were recruited from two universities and their affiliate community colleges in Hong Kong; one national university in Shenzhen, China; two universities in Taipei and Taichung, Taiwan; and one university in Okayama Prefecture, Japan. Ethical approvals were obtained from the participating universities in Hong Kong (as principal site for Hong Kong and Shenzhen), Taiwan, and Japan. University students aged 18-24 years studying in a fulltime undergraduate degree program were eligible to participate. Students were recruited by convenience sampling; information about the study was distributed across campuses through flyers, mass emails, student social media networks, or during class. Interested participants entered a survey website to provide data anonymously through MySurvey v1.1 [39]. The participant information on the survey website clearly stated that participants were providing implied consent by completing the survey.

To prevent repeat entries by the same respondent, the survey website was set to preclude multiple entry with the same electronic device. Between April 2017 and July 2018 , the study website received a total of 2465 hits. Among them, 1346 provided responses to all ACEs and mental health measures (i.e., a completion rate of $54.60 \%$ ); minimal data missing at random $(<1 \%)$ were imputed using mean/mode substitution given the negligible amount of missingness [40]. Of these 1346 respondents, 428 respondents were from Hong Kong $(31.80 \%), 340$ from China (25.26\%), 254 from Taiwan (18.87\%), and 324 from Japan $(24.07 \%)$. The mean age of participants was 20 years $(\mathrm{SD}=1.55)$; over half $(67.90 \%)$ were females. The survey took an average of $27 \mathrm{~min}$ to complete. 


\section{Study measures}

Exposure to childhood adversities was measured using the 29-item WHO Adverse Childhood Experiences-International Questionnaire (ACE-IQ) [29]. This measure assesses exposure to 13 categories of childhood adversities, i.e., physical abuse; sexual abuse; emotional abuse; physical neglect; emotional neglect; domestic violence; household member with mental illness; household member incarceration; household member who was a substance abuser; parental death or separation, bullying, community violence; and collective violence. Details of the Chinese translation, adjusted scoring method, and initial validation of the ACEIQ with the Hong Kong sample are reported elsewhere [9]. The Japanese version followed forward and backward translation protocols per WHO guidelines [41]. Each of the 13 categories of ACEs was dichotomized into "exposed" and "not exposed." The total number of exposures were summed to create an ACE score ranging from 0 to 13; participants were also dichotomized into "no ACE" and "at least one ACE" to denote their overall exposure. The internal consistencies of the ACE-IQ across the four samples were good, with Cronbach's alpha ranging from 0.79 to 0.82 .

Depression and anxiety were measured using the Hospital Anxiety and Depression Scale [42], which was previously validated in Chinese and Japanese youth samples [43, 44]. Depression and anxiety were each measured by seven items on a four-point Likert scale ranging from " 0 , not at all" to " 3 , most of the time"; a higher summed score denotes greater distress. The internal consistencies of the depression and anxiety scales were acceptable for the four samples in this study, with Cronbach's alphas ranging from 0.67 to 0.75 and 0.77 to 0.84 , respectively.

Psychological maladjustment was measured using the Adjustment Disorder-New Module 20 (ADNM-20) [45]. This instrument first asks the respondent to identify the stressful critical life events that occurred in the past year. Then, 20 items were used to measure symptoms of preoccupation with the stressor, failure to adapt, avoidance, depression, anxiety, impulsivity, and functional impairment as a result of those events on a four-point Likert scale ranging from " 1 , never" to " 4 , often." Higher summed scores denote higher maladjustment symptomatology. The Chinese and Japanese versions followed WHO guidelines [41] for forward and backward translation. The internal consistencies of ADNM-20 across the four samples ranged from 0.92 to 0.95 .

Posttraumatic stress was measured using the International Trauma Questionnaire (ITQ) [46], which was developed to assess the self-reports of posttraumatic stress disorder (PTSD) and complex PTSD (CPTSD) according to the diagnostic formulations specified in the recently published ICD-11. The ITQ measures 12 core PTSD/ CPTSD items and 6 items on functional impairment using a five-point Likert scale ranging from " 0 , not at all" to " 4 , extremely." Results of the Chinese translation and initial validation of the ITQ using the Hong Kong sample are reported elsewhere [47]. The Japanese version followed WHO guidelines [41] for forward and backward translation. Diagnostic criteria for PTSD require a score of 2 or above for at least one of the two items from each PTSD symptom cluster (Re-experiencing, Avoidance, and Sense of Threat), and endorsement of at least one item on functional impairment. Diagnostic criteria for CPTSD requires meeting PTSD diagnosis, and scoring 2 or above for at least one of the two items from each symptom cluster that reflect "disturbances in self-organization" (i.e., Affective Dysregulation, Negative Self-Concept, and Disturbed Relationships) and endorsement of at least one item on functional impairment associated with these symptoms. Since the organizing principles of the ICD-11 stipulate that a person may be diagnosed with PTSD or CPTSD, but not both, participants were dichotomized into "PTSD/ CPTSD" and "no trauma diagnosis." The internal consistencies of the two subscales were good across the four samples, ranging from 0.84 to 0.89 for the PTSD items and 0.86-0.90 for the CPTSD items.

Demographic information on participant age and gender was also assessed and served as control variables.

\section{Statistical analysis}

Data analysis was performed using STATA SE14.1 [48]. First, participant age, gender, ACE exposure/score, and mental health outcomes in the full sample were described using summary statistics. Then, latent class analysis was performed using the Penn State LCA Stata Plugin [49] to identify patterns of ACE exposure based on the dichotomized responses to the 13 ACE categories (i.e., "exposed" and "not exposed"). ACE categories were entered into 1-, 2-, 3-, 4-, and 5-class models in an incremental manner. The best model was identified based on substantive interpretation and multiple fit statistics, including Akaike's Information Criteria (AIC) [50], Bayesian Information Criteria (BIC) [51], sample-size adjusted BIC (ssaBIC) [52], Entropy [53], and $p$ value for bootstrap likelihood ratio tests (BSLRT) [54]. Most-likely class membership [55] was used to assign participants to their most representative class; bivariate associations between class membership with ACE exposure/score and mental health variables were assessed using Chi-square or ANOVA tests. Finally, multiple linear or logistic regressions using robust clustering by region (i.e., Hong Kong, China, Taiwan, and Japan) were performed to predict each mental health outcome, controlling for participant age and gender. 


\section{Results}

Descriptive statistics of the full sample are presented in Table 1. The full sample includes 1346 young adults with a mean age of 20 years $(\mathrm{SD}=1.55) ; 67.90 \%(n=914)$ were female and $66.27 \%(n=892)$ reported at least one ACE. On average, these participants were exposed to between 1 and 2 ACEs $(M=1.51, \mathrm{SD}=1.63)$, and scored 5.54 $(\mathrm{SD}=3.44)$ on depression, $7.83(\mathrm{SD}=3.63)$ on anxiety, and 40.03 ( $\mathrm{SD}=13.06)$ on psychological maladjustment. Approximately $6 \%$ of participants $(n=75)$ met diagnostic criteria for PTSD/CPTSD. Figure 1 shows the prevalence of ACE exposure by category. The most commonly reported ACE was physical abuse $(28.08 \%, n=378)$, followed by domestic violence $(23.33 \%, n=314)$ and parental death or separation $(19.24 \%, n=259)$. The LCA model fit statistics are displayed in Table 2. Although the four-class model demonstrated better fit based on AIC and BSLRT results, two of the four classes were represented by less
Table 1 Demographic data and mental health outcomes for full sample and by class

\begin{tabular}{lcccc}
\hline & $\begin{array}{l}\text { Full sample } \\
(n=1346,100.00 \%)\end{array}$ & $\begin{array}{l}\text { Class 1: Low ACEs } \\
(n=1023,76.00 \%)\end{array}$ & $\begin{array}{l}\text { Class 2: } \\
\text { Household } \\
\text { Violence } \\
(n=277,\end{array}$ & $\begin{array}{l}\text { Class 3: House- } \\
\text { hold Dysfunction } \\
(n=46,3.42 \%)\end{array}$ \\
& & & $20.58 \%)$ & \\
\hline Mean age in years (SD) & $20.00(1.55)$ & $19.94(1.53)$ & $20.12(1.59)$ & $20.65(1.65)$ \\
Female $(n, \%)$ & $914(67.90)$ & $700(68.43)$ & $188(67.87)$ & $26(56.52)$ \\
Region $(n, \%)$ & & & & \\
Hong Kong & $428(31.80)$ & $279(27.27)$ & $139(50.18)$ & $10(21.74)$ \\
China & $340(25.26)$ & $285(27.86)$ & $45(26.25)$ & $10(21.74)$ \\
Taiwan & $254(18.87)$ & $194(18.96)$ & $42(15.15)$ & $18(39.13)$ \\
Japan & $324(24.07)$ & $265(25.90)$ & $51(18.41)$ & $8(17.39)$ \\
At least one ACE* $(n, \%)$ & $892(66.27)$ & $569(55.62)$ & $277(100.00)$ & $46(100.00)$ \\
ACE score* (SD) & $1.51(1.63)$ & $0.79(0.85)$ & $3.56(1.29)$ & $5.07(1.51)$ \\
Depression* (SD) & $5.54(3.44)$ & $5.27(3.30)$ & $6.50(3.78)$ & $5.74(3.34)$ \\
Anxiety* (SD) & $7.83(3.63)$ & $7.41(3.45)$ & $9.29(3.89)$ & $8.59(3.80)$ \\
Maladjustment* (SD) & $40.03(13.06)$ & $38.39(12.52)$ & $45.73(13.37)$ & $42.33(13.42)$ \\
PTSD/CPTSD* $(n, \%)$ & $75(5.57)$ & $35(3.42)$ & $33(11.91)$ & $7(15.22)$ \\
\hline
\end{tabular}

*Significant difference across classes at $p<0.01$ from ANOVA or Chi-square tests

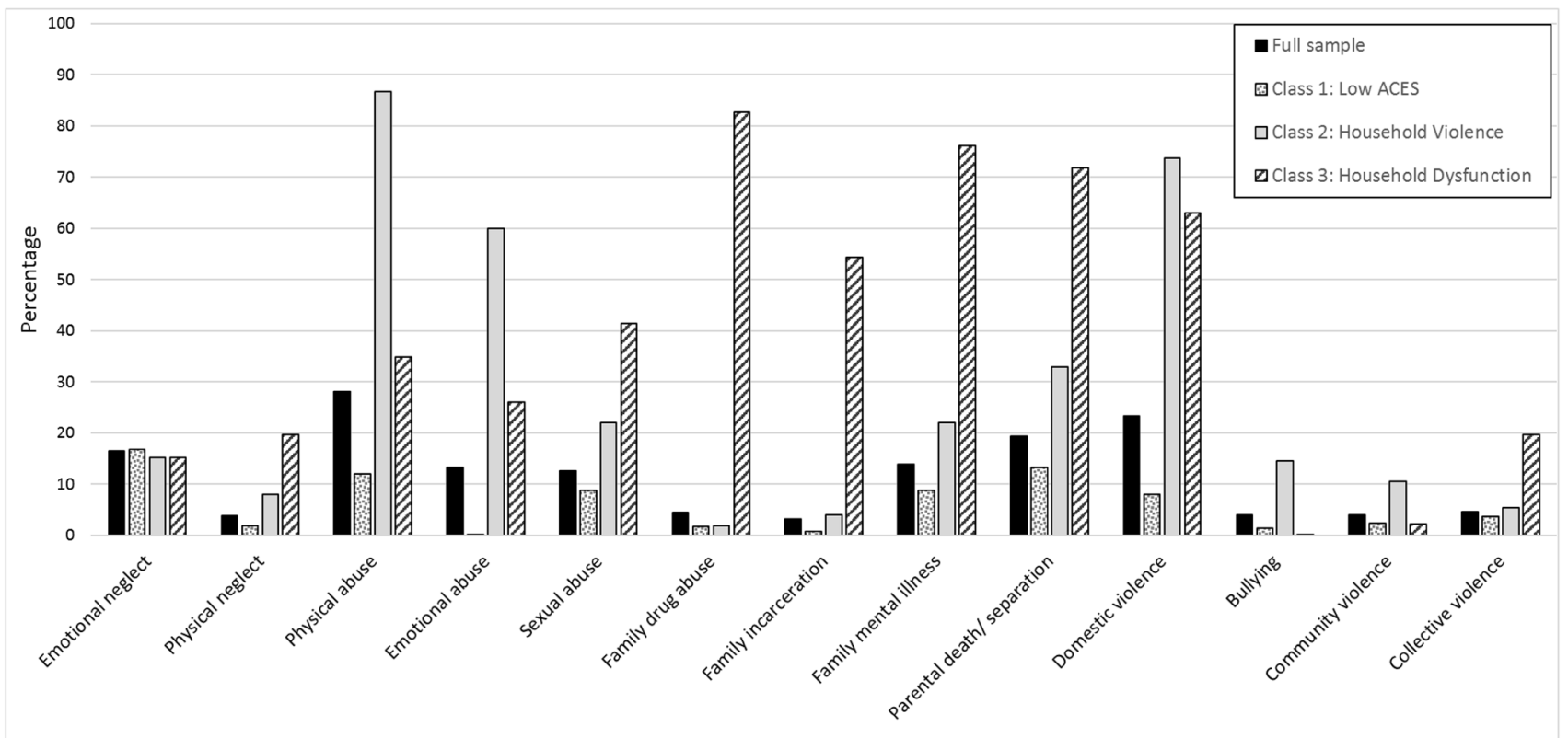

Fig. 1 Percentage of participants exposed to each ACE in full sample and across three classes 
Table 2 Fit indices for latent class analysis for $1-5$ models

\begin{tabular}{llrllll}
\hline Class & Log likelihood & \multicolumn{1}{l}{ AIC } & \multicolumn{1}{l}{ BIC } & ssaBIC & Entropy & BSLRT $p$ value \\
\hline 1 & -5716.38 & 1852.35 & 1920.02 & 1878.72 & - & - \\
2 & -5309.41 & 1066.40 & 1206.93 & 1121.17 & 0.77 & 0.01 \\
3 & $\mathbf{- 5 2 4 0 . 2 3}$ & $\mathbf{9 5 6 . 0 5}$ & $\mathbf{1 1 6 9 . 4 5}$ & $\mathbf{1 0 3 9 . 2 1}$ & $\mathbf{0 . 8 5}$ & $\mathbf{0 . 0 1}$ \\
4 & -5214.14 & 931.86 & 1218.13 & 1043.42 & 0.83 & 0.01 \\
5 & -5206.23 & 944.06 & 1303.20 & 1084.01 & 0.76 & 1.0 \\
\hline
\end{tabular}

Final model selected is in bold

AIC Akaike information criteria, BICBayesian information criteria, ssaBIC sample-size adjusted BIC, $B S L R T$ bootstrapped likelihood ratio test than 50 participants. Furthermore, prior LCA simulations showed that BIC and ssaBIC values are the superior criteria statistics for selecting the best class structure [54]. Therefore, the three-class model was selected based on parsimony, lowest BIC and ssaBIC values, and best precision in model classification (i.e., highest entropy).

Figure 2 depicts the item-response probabilities of each ACE for the three classes. The largest class was labeled Class 1: Low ACEs, and represents the majority of participants $(76.00 \%, n=1023)$. Participants in this class had low likelihood of endorsement across all ACE categories $(<0.20)$. The second largest class was labeled Class 2: Household Violence, and represents $20.58 \%(n=277)$ of the participants. This class had the highest probabilities of endorsing exposures to physical abuse (0.81), emotional abuse (0.54), and witnessing domestic violence (0.68), and relatively low probabilities of endorsing other ACEs $(<0.30)$. The final class represents a minority of the participants $(3.42 \%, n=46)$, and was labeled Class 3: Household
Dysfunction. Participants in this class had high probabilities of endorsing ACEs related to serious household dysfunction, including family member substance use (0.73), incarceration (0.50), and mental illness (0.73); parental separation or death (0.69); and witnessing domestic violence (0.61). Participants in this class also had higher probabilities of endorsing exposure to physical neglect (0.20), sexual abuse (0.38), and collective violence (0.17) compared with other classes. The observed rates of exposure by ACE category in each class are displayed in Fig. 1.

Bivariate analyses show that ACE exposure/score, depression, anxiety, maladjustment, and proportion meeting diagnosis for PTSD/CPTSD significantly differed by class membership (see Table 1). No significant difference in age, gender, or region was observed across classes. Multiple regression analyses show that class membership significantly predicted all mental health outcomes. As shown in Table 3, those in Class 2: Household Violence reported significantly higher depression and maladjustment symptoms compared

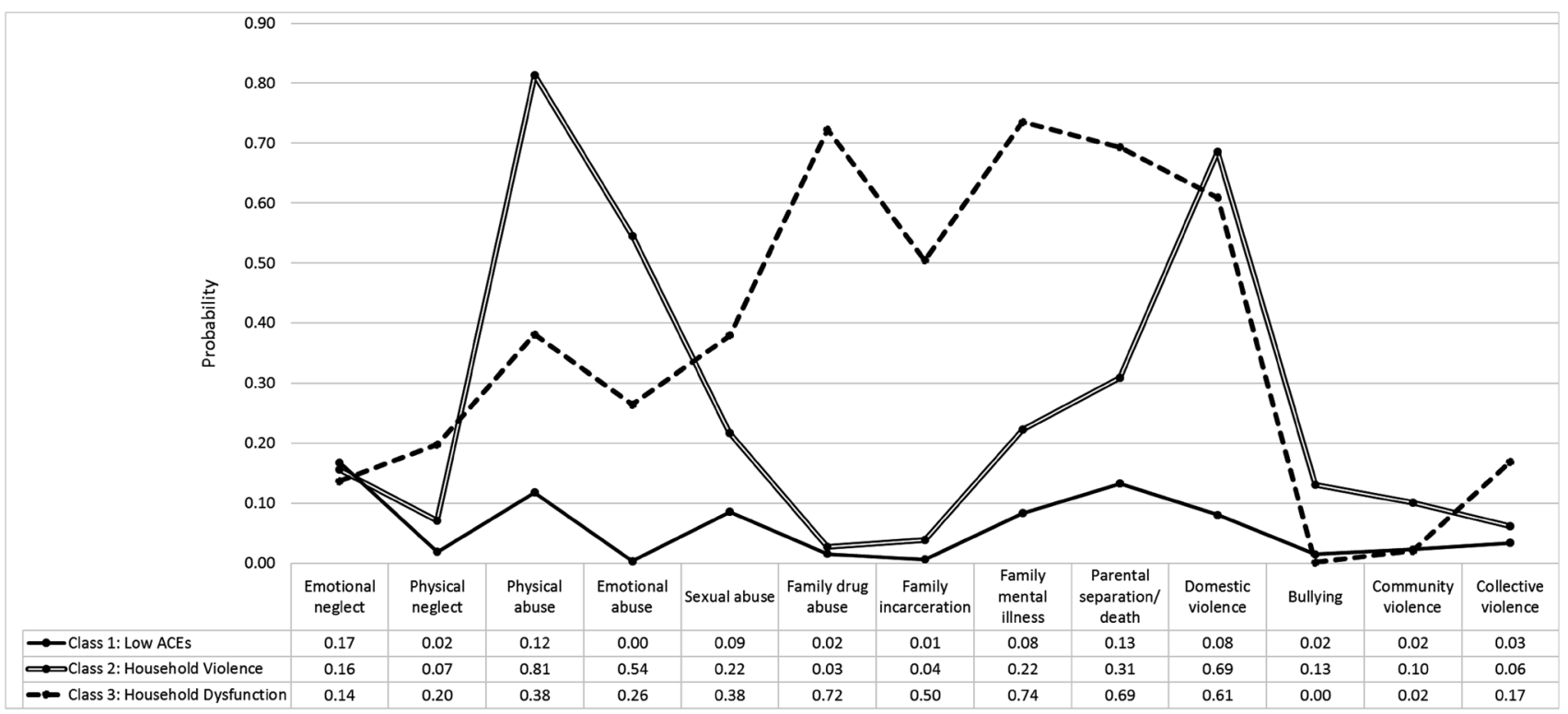

Fig. 2 Item-response probabilities for 13 ACEs across three classes 
Table 3 Multiple regression analyses predicting mental health outcomes by class membership $(n=1346)$

\begin{tabular}{|c|c|c|c|c|}
\hline Predictors & Depression coefficient $(95 \% \mathrm{CI})$ & Anxiety coefficient $(95 \%$ CI) & $\begin{array}{l}\text { Maladjustment } \\
\text { coefficient }(95 \% \text { CI) }\end{array}$ & $\begin{array}{l}\text { PTSD/CPTSD } \\
\text { odds ratio }(95 \% \mathrm{CI})\end{array}$ \\
\hline \multicolumn{5}{|l|}{ Class (Ref: Class 1: Low ACEs) } \\
\hline Class 2: Household Violence & $1.23 *(0.10,2.36)$ & $1.88 * *(1.19,2.57)$ & $7.26 *(3.33,11.20)$ & $3.75^{* *}(2.65,5.31)$ \\
\hline Class 3: Household Dysfunction & $0.43(-1.50,2.36)$ & $1.18 * *(0.68,168)$ & $3.84(-2.88,10.56)$ & $4.74 * *(4.30,5.23)$ \\
\hline Age & $-0.04(-0.43,0.36)$ & $-0.02(-0.34,0.29)$ & $0.62 *(0.09,1.15)$ & $1.12(0.96,1.30)$ \\
\hline Gender (Ref: Male) & $-0.54(-1.98,0.91)$ & $-0.13(-0.73,0.46)$ & $2.74 * *(1.37,4.12)$ & $1.11(0.87,1.42)$ \\
\hline
\end{tabular}

All analyses adjusted for clustering by region (i.e., Hong Kong, China, Taiwan, and Japan); 95\% CI, 95\% confidence interval

*Significant at $p<0.05 ; * *$ significant at $p<0.01$

with those in Class 1: Low ACEs, controlling for age and gender; no significant differences were found between those in Class 1: Low ACEs and Class 3: Household Dysfunction. Separately, both Class 2: Household Violence and Class 3: Household Dysfunction had significantly higher anxiety symptoms and odds for meeting diagnostic criteria for PTSD/CPTSD, controlling for age and gender, compared with Class 1: Low ACEs. In particular, the odds for screening positive for PTSD/CPTSD was higher for those in Class 3: Household Dysfunction $(\mathrm{OR}=4.74, \mathrm{SE}=0.24)$ than those in Class 2: Household Violence $(\mathrm{OR}=3.75, \mathrm{SE}=0.66)$ when compared with those in Class 1: Low ACEs.

\section{Discussion}

The present findings corroborate with our previous study [9], and validate a distinct pattern of ACE exposure in East Asia marked by exposures to multiple forms of household violence (i.e., physical abuse, emotional abuse, and witnessing domestic violence) in the absence of other serious household dysfunction. Although our results were not generated from a representative sample, the proportion of young adults endorsing this ACE pattern $(20.58 \%)$ were similar to that reported in a representative survey of 1093 Hong Kong children, where $18.1 \%$ reported co-occurring child physical abuse and severe intimate partner violence in the household [56]. Another study of dual victimization to interparental violence and physical abuse in a national survey of Taiwanese college students reported a co-occurrence rate of $11.3 \%$ [38], which corresponds with the comparatively lower rates of young adults from Taiwan representing this class $(15.15 \%)$. Nonetheless, our results demonstrated that growing up in violent but otherwise well-functioning households is a relatively common experience among children in East Asia. It is possible that culturally ingrained values and beliefs, such as patriarchal norms, endorsement of physical punishment, and normalization of family violence, may increase children's exposure to both direct and indirect forms of violence at home $[20,57,58]$, and potentiate the risk for this unique pattern of ACE exposure in this cultural group.

Separately, we uncovered an ACE pattern with high probabilities for multiple serious household dysfunction (i.e., Class 3), akin to those reported in other pattern-based ACE studies using Western samples [7, 25]. However, the proportion of young adults represented by this class was relatively small $(3.42 \%)$, supporting prior assertions that non-violencebased ACEs in more conservative Asian cultures are relatively rare [30]. Furthermore, young adults representing this class were also exposed to a significantly higher number of ACEs on average compared with those in other classes (i.e., ACE score of 5.07 versus 0.79 for Class 1 and 3.56 for Class 2), indicating that serious household dysfunctions, although rare, likely co-occur and heighten risks for other forms of ACEs. Importantly, we found that despite having a higher ACE score, those in Class 3: Household Dysfunction appeared to fare better in terms of their mental health than those in Class 2: Household Violence (except posttraumatic stress) when compared with those in Class 1: Low ACEs. This finding contradicts recent work demonstrating latent class and cumulative risk approaches provide largely similar outcomes predictions among US young adults [25], and provides further support that young adults' mental health, at least under certain contexts, are more closely linked with the nature and pattern of ACE co-occurrence rather than the number of ACEs [34].

Our study was the first to associate different mental health outcomes with unique ACE patterns among young adults in East Asia. We observed that mental health outcomes significantly differed across classes, but demographic variables (i.e., age, gender, and region) did not. Specifically, those in Class 2: Household Violence reported higher depression and maladjustment symptoms compared with those with low ACEs, but this was not the case for those in Class 3: Household Dysfunction. These findings support prior work, demonstrating that certain types of ACEs (i.e., child maltreatment) may individually contribute more adverse mental health outcomes compared with other ACEs (e.g., serious household dysfunctions) [7, 59]. Indeed, one study found 
that physical abuse or neglect by a caregiver had the strongest independent association with depression among other forms of victimization or adversities [3]. In the context of witnessing domestic violence, which was unequivocally high across Classes 2 and 3, prior studies demonstrated that depression and maladjustment symptoms were significantly higher among those who also felt personally threatened by the violence (e.g., physical abuse or other child-directed aggression) $[38,60,61]$. On the other hand, anxiety and posttraumatic stress symptoms were higher in both Classes 2 and 3 when compared with those with low ACEs (Class 1). These findings correspond with prior research suggesting ACEs, regardless of their presentation, universally tap into fear and danger, and serve as the earliest roots of developmental psychopathology for fear-related disorders [62]. Furthermore, perceived threat and control from chronic and multiple ACEs have been associated with greater odds for clinically significant levels of posttraumatic stress in children [60]. Together, our findings point to how typologies of ACE exposure vary by context, and may differentially impact mental health outcomes by early adulthood.

Several study limitations are noted. First, the generalizability of our findings was limited by the convenience nature of participant sampling and a 55\% completion rate among those who entered the survey weblink; the study samples across regions were also largely homogenous (i.e., educated young people enrolled in an undergraduate degree program). It is possible that sampling from other community-based or clinical settings may uncover additional patterns of ACE exposure. Second, although an anonymous online survey format was used, retrospective self-reports of ACEs may still be biased from inaccurate recall or social desirability among young adults [63]. Nonetheless, emerging evidence shows that the associations between ACEs and reported health outcomes found using retrospective data can be confirmed with prospective data [64]. We also did not account for age of onset or duration of different ACEs, or exposure to trauma after age 18, which may have significant impacts on mental health by early adulthood. Third, our cross-sectional study design precluded understanding how psychopathology may develop or change over time. Finally, instruments employed in Japan were not formally validated, but we translated the survey items with strict adherence to published guidelines.

In spite of the above limitations, our observation that different types of adverse mental health outcomes are associated with different patterns of ACEs in young East Asian adults has three important implications. First, our findings suggest that ACE screening should focus on the ACE patterns rather than the number of ACEs. This is an important finding, because many proposed ACE screening programs for children were designed to generate a total ACE score, and typically involve assessing symptoms or reactions to traumatic stress in relation to their cumulative exposure
$[65,66]$. The early identification of certain ACE exposure patterns in children may help to predict the risk of developing specific mental disorders in young adulthood, and hence afford an opportunity to identify children that would benefit from targeted early interventions. This may be particularly useful in cases where children have not yet exhibited obvious reactions to their ACEs, as there is often a significant time lag between identification, referral, and receiving appropriate treatment for posttraumatic stress or other mental health disorders [67].

Second, prevention and intervention approaches to mitigate the negative consequences of ACEs need to be specific to context and culture. For example, family-based interventions that aim to improve adaptive family functioning and strengthen the individual resilience of young people have shown to mitigate the negative outcomes of ACE exposure on mental health in adulthood [68]. These intervention targets may have higher therapeutic potential in cultures that emphasize preserving family harmony and protecting family honor, such as East Asia [38]. Furthermore, our results support that exposure to multiple and often co-occurring forms of household dysfunctions does not necessarily lead to poorer mental health outcomes; prior research also suggests this to be the case if the childhood holding environment remains positive [59]. This is particularly salient for collectivistic societies, such as East Asia, where children from dysfunctional households may still receive quality care from extended kinship or neighbors in the wider community [69]. Thus, these positive cultural tenets could also form the basis for wider community-based preventative and intervention strategies to foster positive child development and resilience building in the context of ACEs.

Finally, the findings from our study might be useful for promoting public awareness of the mental health consequences of ACE exposure in East Asian populations. This information may be of particular relevance in this cultural setting, where the magnitude of the long-term negative effects of household violence are not fully recognized due to the normalization and trivialization of physical assault and emotional aggression in the home [20,70]. More culture-based research is needed to further understand ACE patterns across settings and their associated outcomes to tailor culturally congruent and effective approaches to support those exposed to adversities early in life. To this end, given the rich history of East Asian diasporas and increasing globalization, future research should also explore whether and how ACE exposure and its identification and mitigation may differ for immigrant and non-immigrant Asian communities.

Acknowledgements This study was supported by The Hong Kong Polytechnic University Internal Start-Up Fund. 


\section{Compliance with ethical standards}

Conflict of interest On behalf of all authors, the corresponding author states that there is no conflict of interest.

\section{References}

1. Felitti VJ, Anda RF, Nordenberg D, Williamson DF, Spitz AM, Edwards V, Koss MP, Marks JS (1998) Relationship of childhood abuse and household dysfunction to many of the leading causes of death in adults. The Adverse Childhood Experiences (ACE) Study. Am J Prev Med 14(4):245-258

2. Green JG, McLaughlin KA, Berglund PA, Gruber MJ, Sampson NA, Zaslavsky AM, Kessler RC (2010) Childhood adversities and adult psychiatric disorders in the national comorbidity survey replication I: associations with first onset of DSM-IV disorders. Arch Gen Psychiatry 67(2):113-123. https://doi.org/10.1001/ archgenpsychiatry.2009.186

3. Turner HA, Finkelhor D, Ormrod R (2006) The effect of lifetime victimization on the mental health of children and adolescents. Soc Sci Med 62(1):13-27. https://doi.org/10.1016/j.socsc imed.2005.05.030

4. Kessler RC, McLaughlin KA, Green JG, Gruber MJ, Sampson NA, Zaslavsky AM, Aguilar-Gaxiola S, Alhamzawi AO, Alonso J, Angermeyer M, Benjet C, Bromet E, Chatterji S, de Girolamo G, Demyttenaere K, Fayyad J, Florescu S, Gal G, Gureje O, Haro JM, Hu CY, Karam EG, Kawakami N, Lee S, Lepine JP, Ormel J, Posada-Villa J, Sagar R, Tsang A, Ustun TB, Vassilev S, Viana MC, Williams DR (2010) Childhood adversities and adult psychopathology in the WHO World Mental Health Surveys. Br J Psychiatry 197(5):378-385. https://doi.org/10.1192/bjp.bp.110.08049 9

5. Hughes K, Bellis MA, Hardcastle KA, Sethi D, Butchart A, Mikton C, Jones L, Dunne MP (2017) The effect of multiple adverse childhood experiences on health: a systematic review and meta-analysis. Lancet Public health 2(8):e356-e366. https://doi. org/10.1016/s2468-2667(17)30118-4

6. Cavanaugh CE, Petras H, Martins SS (2015) Gender-specific profiles of adverse childhood experiences, past year mental and substance use disorders, and their associations among a national sample of adults in the United States. Soc Psychiatry Psychiatr Epidemiol 50(8):1257-1266. https://doi.org/10.1007/s0012 7-015-1024-3

7. Shin SH, McDonald SE, Conley D (2018) Patterns of adverse childhood experiences and substance use among young adults: a latent class analysis. Addict Behav 78:187-192. https://doi. org/10.1016/j.addbeh.2017.11.020

8. Roos LE, Afifi TO, Martin CG, Pietrzak RH, Tsai J, Sareen J (2016) Linking typologies of childhood adversity to adult incarceration: findings from a nationally representative sample. Am J Orthopsychiatry 86(5):584-593. https://doi.org/10.1037/ort00 00144

9. Ho GWK, Chan ACY, Chien W-T, Bressington DT, Karatzias T (2019) Examining patterns of adversity in Chinese young adults using the Adverse Childhood Experiences-International Questionnaire (ACE-IQ). Child Abuse Negl 88:179-188. https://doi. org/10.1016/j.chiabu.2018.11.009

10. Bellis MA, Hughes K, Leckenby N, Jones L, Baban A, Kachaeva M, Povilaitis R, Pudule I, Qirjako G, Ulukol B, Raleva M, Terzic N (2014) Adverse childhood experiences and associations with health-harming behaviours in young adults: surveys in eight eastern European countries. Bull World Health Organ 92(9):641-655. https://doi.org/10.2471/blt.13.129247
11. Marginson S (2011) Higher education in East Asia and Singapore: rise of the Confucian model. High Educ 61(5):587-611. https:// doi.org/10.1007/s10734-010-9384-9

12. Fujiwara T, Kawakami N (2011) Association of childhood adversities with the first onset of mental disorders in Japan: results from the World Mental Health Japan, 2002-2004. J Psychiatr Res 45(4):481-487. https://doi.org/10.1016/j.jpsychires.2010.08.002

13. Kim YH (2017) Associations of adverse childhood experiences with depression and alcohol abuse among Korean college students. Child Abuse Negl 67:338-348. https://doi.org/10.1016/j. chiabu.2017.03.009

14. Tran QA, Dunne MP, Vo TV, Luu NH (2015) Adverse childhood experiences and the health of university students in eight provinces of Vietnam. Asia Pac J Public Health 27(8 Suppl):26S-32S. https://doi.org/10.1177/1010539515589812

15. Wei W (2013) Adverse childhood experience as a risk factor for adolescent pregnancy in China. Int J Child Adolesc Health $6(3): 323$

16. Li Y, Cao F, Cao D, Liu J (2015) Nursing students' post-traumatic growth, emotional intelligence and psychological resilience. J Psychiatr Ment Health Nurs 22(5):326-332

17. Chang X, Jiang X, Mkandarwire T, Shen M (2019) Associations between adverse childhood experiences and health outcomes in adults aged 18-59 years. PLoS one 14(2):e0211850. https://doi. org/10.1371/journal.pone.0211850

18. Shwalb DW, Nakazawa J, Yamamoto T, Hyun J-H (2004) Fathering in Japanese, Chinese, and Korean cultures: a review of the research literature. In: Wiley John, Inc Sons (eds) The role of the father in child development, 4th edn. Hoboken, New York, pp 146-181

19. Douglas EM (2006) Familial violence socialization in childhood and later life approval of corporal punishment: a cross-cultural perspective. Am J Orthopsychiatry 76(1):23-30

20. Ozaki R, Otis MD (2016) Gender equality, patriarchal cultural norms, and perpetration of intimate partner violence: comparison of male university students in Asian and European cultural contexts. Violence Against Women 23(9):1076-1099. https://doi. org/10.1177/1077801216654575

21. Coleman DL, Dodge KA, Campbell SK (2010) Where and how to draw the line between reasonable corporal punishment and abuse. Law Contemp Probl 73(2):107-166

22. Ho GWK, Gross DA (2015) Differentiating physical discipline from abuse: $Q$ findings from Chinese American mothers and pediatric nurses. Child Abuse Negl 43:83-94. https://doi. org/10.1016/j.chiabu.2015.03.012

23. Barboza GE (2018) Latent classes and cumulative impacts of adverse childhood experiences. Child Maltreatment 23(2):111125. https://doi.org/10.1177/1077559517736628

24. McLafferty M, Armour C, McKenna A, O'Neill S, Murphy S, Bunting B (2015) Childhood adversity profiles and adult psychopathology in a representative Northern Ireland study. J Anxiety Disord 35:42-48. https://doi.org/10.1016/j.janxdis.2015.07.004

25. Merians AN, Baker MR, Frazier P, Lust K (2019) Outcomes related to adverse childhood experiences in college students: comparing latent class analysis and cumulative risk. Child Abuse Negl 87:51-64. https://doi.org/10.1016/j.chiabu.2018.07.020

26. Charak R, Koot HM (2015) Severity of maltreatment and personality pathology in adolescents of Jammu, India: a latent class approach. Child Abuse Negl 50:56-66. https://doi.org/10.1016/j. chiabu.2015.05.010

27. Chng GS, Li D, Chu CM, Ong T, Lim F (2018) Family profiles of maltreated children in Singapore: a latent class analysis. Child Abuse Negl 79:465-475. https://doi.org/10.1016/j.chiab u.2018.02.029

28. Yang FAN, Lou VWQ (2015) Childhood adversities, urbanisation and depressive symptoms among middle-aged and older 
adults: evidence from a national survey in China. Ageing Soc 36(5):1031-1051. https://doi.org/10.1017/S0144686X150002 39

29. World Health Organization (2016) Adverse childhood experiences international questionnaire (ACE-IQ). www.who.int/violence_ injury_prevention/violence/activities/adverse_childhood_exper iences

30. Choo W-Y, Dunne MP, Marret MJ, Fleming M, Wong Y-L (2011) Victimization experiences of adolescents in Malaysia. J Adolesc Health 49(6):627-634. https://doi.org/10.1016/j.jadohealth .2011 .04 .020

31. Stoltenborgh M, Bakermans-Kranenburg MJ, van Ijzendoorn $\mathrm{MH}$, Alink LRA (2013) Cultural-geographical differences in the occurrence of child physical abuse? A meta-analysis of global prevalence. Int J Psychol 48(2):81-94. https://doi.org/10.1080/00207 594.2012.697165

32. Herringa RJ, Birn RM, Ruttle PL, Burghy CA, Stodola DE, Davidson RJ, Essex MJ (2013) Childhood maltreatment is associated with altered fear circuitry and increased internalizing symptoms by late adolescence. Proc Natl Acad Sci 110(47):19119-19124. https://doi.org/10.1073/pnas.1310766110

33. Schilling EA, Aseltine RH Jr, Gore S (2007) Adverse childhood experiences and mental health in young adults: a longitudinal survey. BMC Public Health 7:30. https://doi. org/10.1186/1471-2458-7-30

34. Schilling EA, Aseltine RH, Gore S (2008) The impact of cumulative childhood adversity on young adult mental health: measures, models, and interpretations. Soc Sci Med 66(5):1140-1151. https ://doi.org/10.1016/j.socscimed.2007.11.023

35. Berzenski SR, Yates TM (2011) Classes and consequences of multiple maltreatment: a person-centered analysis. Child Maltreatment 16(4):250-261. https://doi.org/10.1177/1077559511428353

36. Miller-Graff LE, Howell KH, Martinez-Torteya C, Hunter EC (2015) Typologies of childhood exposure to violence: associations with college student mental health. J Am Coll Health 63(8):539549. https://doi.org/10.1080/07448481.2015.1057145

37. Han M, Choi Y, Jung S (2015) Exploring the relationship between exposure to interparental violence and child physical abuse in childhood and the impacts on mental health problems in later young adulthood among South Korean college students. Int Soc Work 59(6):821-835. https://doi.org/10.1177/0020872814562481

38. Shen AC-T (2009) Long-term effects of interparental violence and child physical maltreatment experiences on PTSD and behavior problems: a national survey of Taiwanese college students. Child Abuse Negl 33(3):148-160. https://doi.org/10.1016/j.chiab u.2008.07.006

39. The Hong Kong Polytechnic University (2016) mySurvey (Version 1.1) [Software]. https://www.polyu.edu.hk/mysurvey/

40. Saunders JA, Morrow-Howell N, Spitznagel E, Doré P, Proctor EK, Pescarino R (2006) Imputing missing data: a comparison of methods for social work researchers. Soc Work Res 30(1):19-31

41. World Health Organization (2017) Process of translation and adaptation of instruments. http://www.who.int/substance_abuse /research_tools/translation/en/

42. Zigmond AS, Snaith RP (1983) The hospital anxiety and depression scale. Acta Psychiatr Scand 67(6):361-370

43. Chan YF, Leung DY, Fong DY, Leung CM, Lee AM (2010) Psychometric evaluation of the hospital anxiety and depression scale in a large community sample of adolescents in Hong Kong. Qual Life Res 19(6):865-873. https://doi.org/10.1007/s1113 6-010-9645-1

44. Matsudaira T, Igarashi H, Kikuchi H, Kano R, Mitoma H, Ohuchi K, Kitamura T (2009) Factor structure of the Hospital Anxiety and Depression Scale in Japanese psychiatric outpatient and student populations. Health Qual Life Outcomes 7(1):42. https://doi. org/10.1186/1477-7525-7-42
45. Glaesmer H, Romppel M, Brahler E, Hinz A, Maercker A (2015) Adjustment disorder as proposed for ICD-11: dimensionality and symptom differentiation. Psychiatry Res 229(3):940-948. https:// doi.org/10.1016/j.psychres.2015.07.010

46. Cloitre M, Shevlin M, Brewin CR, Bisson JI, Roberts NP, Maercker A, Karatzias T, Hyland P (2018) The International trauma questionnaire: development of a self-report measure of ICD-11 PTSD and complex PTSD. Acta Psychiatr Scand 138(6):536-546. https://doi.org/10.1111/acps.12956

47. Ho GWK, Karatzias T, Cloitre M, Chan ACY, Bressington D, Chien WT, Hyland P, Shevlin M (2019) Translation and validation of the Chinese ICD-11 international trauma questionnaire (ITQ) for the assessment of posttraumatic stress disorder (PTSD) and complex PTSD (CPTSD). Eur J Psychotraumatol 10(1):1608718. https://doi.org/10.1080/20008198.2019.1608718

48. StataCorp (2015) Stata statistical software: release 14. StataCorp LP, College Station

49. The Methodology Center (2015) LCA stata plugin (Version 1.2) [Software]. Penn State, University Park

50. Akaike H (1987) Factor analysis and AIC. Psychometrika 52(3):317-332. https://doi.org/10.1007/bf02294359

51. Schwarz G (1978) Estimating the dimension of a model. Ann Stat 6(2):461-464

52. Sclove SL (1987) Application of model-selection criteria to some problems in multivariate analysis. Psychometrika 52(3):333-343. https://doi.org/10.1007/bf02294360

53. Magidson J, Vermunt J (2002) Latent class models for clustering: a comparison with K-means. Can J Market Res 20(1):36-43

54. Nylund KL, Asparouhov T, Muthén BO (2007) Deciding on the number of classes in latent class analysis and growth mixture modeling: a Monte Carlo simulation study. Struct Equ Model 14(4):535-569

55. Clark SL, Muthén B (2009) Relating latent class analysis results to variables not included in the analysis. Retrieved from https:// www.statmodel.com/download/relatinglca.pdf

56. Chan KL (2011) Children exposed to child maltreatment and intimate partner violence: a study of co-occurrence among Hong Kong Chinese families. Child Abuse Negl 35(7):532-542. https ://doi.org/10.1016/j.chiabu.2011.03.008

57. Fulu E, Miedema S, Roselli T, McCook S, Chan KL, Haardörfer R, Jewkes R, Fulu E, Jewkes R, Warner X, Miedema S, Roselli T, Lang J, Naved RT, Huque H, Farah S, Shuvra MMR, Erken A, Xiangxian W, Gang F, Hongtao L, Mudrovcic Z, Hua W, Hoekman A, Nikulainen E, Coquelin B, Khan M, Kusuma W, Manero CM, Larsen F, Fulu E, Warner X, Moussavi S, de Mel N, Peiris P, Gomez S, Team SI, Jinadasa K, Jewkes R, Sikweyiya Y, Shai N, Drapuluvik-Tinabar F, Magoola P, Agyenta A, Shanahan T, Vienings T, Jewkes R, Garcia-Moreno C, Naved RT, Jinadasa K, Vienings T, Kusuma W, Jewkes R, Connell R, Barker G, Greig A, Roy R, Verma R, Sen KM, Johnson S (2017) Pathways between childhood trauma, intimate partner violence, and harsh parenting: findings from the UN Multi-country Study on Men and Violence in Asia and the Pacific. Lancet Glob Health 5(5):e512-e522. https ://doi.org/10.1016/S2214-109X(17)30103-1

58. Ji K, Finkelhor D (2015) A meta-analysis of child physical abuse prevalence in China. Child Abuse Negl 43:61-72. https://doi. org/10.1016/j.chiabu.2014.11.011

59. Hovens JGFM, Wiersma JE, Giltay EJ, Van Oppen P, Spinhoven P, Penninx BWJH, Zitman FG (2010) Childhood life events and childhood trauma in adult patients with depressive, anxiety and comorbid disorders vs. controls. Acta Psychiatr Scand 122(1):6674. https://doi.org/10.1111/j.1600-0447.2009.01491.x

60. Spilsbury JC, Belliston L, Drotar D, Drinkard A, Kretschmar J, Creeden R, Flannery DJ, Friedman S (2007) Clinically significant trauma symptoms and behavioral problems in a community-based sample of children exposed to domestic violence. $\mathrm{J}$ 
Family Violence 22(6):487-499. https://doi.org/10.1007/s1089 6-007-9113-z

61. Grych JH, Jouriles EN, Swank PR, McDonald R, Norwood WD (2000) Patterns of adjustment among children of battered women. J Consult Clin Psychol 68(1):84

62. Pynoos RS, Steinberg AM, Piacentini JC (1999) A developmental psychopathology model of childhood traumatic stress and intersection with anxiety disorders. Biol Psychiatry 46(11):1542-1554. https://doi.org/10.1016/S0006-3223(99)00262-0

63. Naicker SN, Norris SA, Mabaso M, Richter LM (2017) An analysis of retrospective and repeat prospective reports of adverse childhood experiences from the South African Birth to Twenty Plus cohort. PLoS One 12(7):e0181522. https://doi.org/10.1371/ journal.pone. 0181522

64. Reuben A, Moffitt TE, Caspi A, Belsky DW, Harrington H, Schroeder F, Hogan S, Ramrakha S, Poulton R, Danese A (2016) Lest we forget: comparing retrospective and prospective assessments of adverse childhood experiences in the prediction of adult health. J Child Psychol Psychiatry 57(10):1103-1112. https://doi. org/10.1111/jcpp.12621

65. Purewal SK, Bucci M, Gutiérrez Wang L, Koita K, Silvério Marques S, Oh D, Burke Harris N (2016) Screening for adverse childhood experiences (ACEs) in an integrated pediatric care model. Zero Three 37(1):10-17

66. Finkelhor D (2018) Screening for adverse childhood experiences (ACEs): cautions and suggestions. Child Abuse Negl 85:174-179. https://doi.org/10.1016/j.chiabu.2017.07.016

67. Lieberman AF, Chu A, Van Horn P, Harris WW (2011) Trauma in early childhood: empirical evidence and clinical implications. Dev Psychopathol 23(2):397-410. https://doi.org/10.1017/s0954 579411000137

68. Oshri A, Lucier-Greer M, O'Neal CW, Arnold AL, Mancini JA, Ford JL (2015) Adverse childhood experiences, family functioning, and resilience in military families: a pattern-based approach. Fam Relat 64(1):44-63. https://doi.org/10.1111/fare.12108

69. Kim UE, Triandis HC, Kâğitçibaşi ÇE, Choi S-CE, Yoon GE (1994) Individualism and collectivism: theory, method, and applications. Sage Publications Inc, Thousand Oaks

70. Leggett A (2017) Online civic engagement and the anti-domestic violence movement in china: shifting norms and influencing law. VOLUNTAS Int J Volunt Nonprofit Org 28(5):2251-2277. https ://doi.org/10.1007/s11266-016-9680-9 